\title{
Eine spieltheoretische Analyse des Länderfinanzausgleichs in der Bundesrepublik Deutschland
}

\author{
Reiner Wolff
}

Accepted: 16 November 2006 / Published online: 18 January 2007

(C) Springer-Verlag 2007

Zusammenfassung Diese Arbeit bildet den bundesdeutschen horizontalen Finanzausgleich in einem kooperativen Spiel zwischen den beteiligten Bundesländern ab. In diesem Spiel können Paare disjunkter Koalitionen von Spielern fiktive Solidargemeinschaften bilden und nach den Bestimmungen des Finanzausgleichsgesetzes einen gegenseitigen Finanzausgleich herbeiführen. Auf dieser Basis gewinnen wir in der Spiellösung hypothetische Ausgleichsbeiträge der jeweiligen Geberländer und Ausgleichszuweisungen an die betreffenden Nehmerländer für den Finanzausgleich der Jahre 1998 und 2002. Die Abweichungen der Spielergebnisse von den gesetzlichen Abschöpfungen und Zuweisungen interpretieren wir als eine verdeckte Progression im Länderfinanzausgleich, die sich überwiegend zu Lasten der Geberländer auswirkt. Der damit verbundene Gesamteffekt auf das Transfervolumen beträgt in beiden Jahren ca. 28\%. Für den neuen Länderfinanzausgleich ab dem Jahre 2005 zeichnen sich vergleichbare Ergebnisse ab.

Schlüsselwörter Finanzausgleich · kooperative Spiele · Shapley-Wert

JEL Klassifikationen C71, H77

\begin{abstract}
The German system of financial equalization among the country's 16 federal states is built into a cooperative game. In this game, pairs of disjoint coalitions of players may form alliances which harmonize tax revenues among its members according to the procedural regulations of the law. The game solution generates a hypothetical transfer scheme for the set of all 16 states with reference to the years 1998
\end{abstract}

R. Wolff $(\bullet)$

Departement für Quantitative Wirtschaftsforschung, Universität Fribourg, Bd de Pérolles 90, CH-1700 Fribourg, Switzerland

e-mail: Reiner.Wolff@unifr.ch 
and 2002. Deviations from the respective empirical schemes are interpreted as a hidden progressivity which comes mainly at the cost of the richer states. The impact on the overall transfer volume amounts to about $28 \%$ in both years. Similar results are to be expected for the new system of financial equalization which started in 2005 .

\section{Problemstellung ${ }^{\dagger}$}

Das Transfervolumen des gesamten bundesstaatlichen Finanzausgleichs der Bundesrepublik Deutschland in den ersten acht Jahren seit der Einbeziehung der neuen Bundesländer (1995-2002) betrug im Jahresdurchschnitt 27.718 Mill. Euro. Davon entfielen im Mittel 6.976 Mill. Euro (25,2\%) auf den horizontalen Finanzausgleich zwischen den Ländern, der auch als Länderfinanzausgleich im engeren Sinne bezeichnet wird. Er soll sicherstellen, dass ,,die unterschiedliche Finanzkraft der Länder angemessen ausgeglichen wird.“ (Artikel 107, Absatz 2, Grundgesetz.) Die übrigen Transfers bestanden durchschnittlich zu etwa einem Drittel aus dem Vorwegausgleich der Umsatzsteuer und zu etwa zwei Dritteln aus Bundesergänzungszuweisungen. Im Ergebnis des horizontalen Finanzausgleichs zwischen den Ländern (im Weiteren kurz: Länderfinanzausgleich) wurden im genannten Zeitraum insgesamt immerhin 55.807 Mill. Euro transferiert. Diese Summe ist nahezu vollständig von den Ländern Baden-Württemberg, Bayern, Hamburg, Hessen und Nordrhein-Westfalen aufgebracht worden. Die weitaus höchsten Einnahmen aus dem Länderfinanzausgleich erzielte in jedem Jahr das Land Berlin. (Vgl. BMF 2004b: 38-46.)

Das hierfür maßgebliche Gesetz über den Finanzausgleich zwischen Bund und Ländern (Finanzausgleichsgesetz - FAG) vom 23. Juni 1993 (zuletzt geändert am 16. Juni 1998) wurde vom Bundesverfassungsgericht bereits am 11. November 1999 zum Übergangsrecht herabgestuft, nachdem die Geberländer Baden-Württemberg, Bayern und Hessen drei Normenkontrollanträge gegen dieses Gesetz gestellt hatten. Der höchstrichterlichen Entscheidung war eine länger andauernde politische Auseinandersetzung zwischen den Ländern über die Leistungsgerechtigkeit und Transparenz des Föderalen Konsolidierungsprogramms (Solidarpakt I) zur Finanzierung der deutschen Einheit vorausgegangen. Im Ergebnis der politischen Beratungen über die Fortführung des Föderalen Konsolidierungsprogramms (Solidarpakt II) wurde der Konflikt zwei Jahre später vom Deutschen Bundestag und vom Bundesrat durch das Maßstäbegesetz vom 9. September 2001 und durch das Finanzausgleichsgesetz vom 20. Dezember 2001 für die Periode 2005-2019 einvernehmlich und insbesondere mit der Zustimmung aller Länder beigelegt.

Der Vorgang illustriert anschaulich, in welchem Ausmaß die politischen Bewertungen des Solidarpakts von der Höhe der Transfers beeinflusst werden, die die neuen Bundesländer im Länderfinanzausgleich zusätzlich auslösen. Aus der Sicht der alten Bundesländer stellen diese Transfers (Grenz-)Kosten dar, die diese Länder je

\footnotetext{
$\dagger$ Den Teilnehmern und Teilnehmerinnen der Jahrestagung 2004 des Ausschusses für Regionaltheorie und -politik des Vereins für Socialpolitik (Kiel, 7.-9. Oktober 2004) bin ich für eine anregende Diskussion zu Dank verpflichtet. Zwei anonymen Gutachtern danke ich ebenfalls für ihre wertvollen Hinweise.
} 
nachdem über eine Erhöhung ihrer Beitragsverpflichtungen oder über eine Reduktion ihrer bisherigen Ausgleichsansprüche tragen müssen. Vor diesem Hintergrund stellen die Normenkontrollanträge der Beschwerdeführer Baden-Württemberg, Bayern und Hessen den Versuch eines Teils der alten Bundesländer dar, einen ehedem bewährten politischen Konsens aufzukündigen und die Kooperation - jedenfalls in der hergebrachten Form - zu verweigern. Darin erkennen wir ein strategisches Element, dem wir in der vorliegenden Arbeit nachgehen wollen.

Hierzu bilden wir den Länderfinanzausgleich in einem kooperativen Spiel ab. In diesem Spiel treten die Länder als Spieler auf, die durch horizontale Ausgleichszahlungen einen solidarischen Beitrag zur Wahrung der ,Einheitlichkeit der Lebensverhältnisse im Bundesgebiet" (Artikel 106, Absatz 3, Grundgesetz) leisten können. Das Finanzausgleichsgesetz soll ein wesentlicher Bestandteil des Spiels sein und nicht etwa durch das Spiel ersetzt werden. Insoweit nehmen wir an, dass das Finanzausgleichsgesetz den Verfassungsauftrag des „,billigen“, d.h. fairen Ausgleichs (Artikel 106, Absatz 3, Grundgesetz) im Grundsatz verbindlich auslegt. Wir verfolgen also nicht wie andere Autoren das Ziel, einen mathematisch möglichst einfachen Ausgleichstarif zu finden, dessen funktionale Form und Parametrisierung plausibel den Verfassungsnormen angepasst ist (z.B. Buhl 1986; Buhl/Pfingsten 1986, 1991; Taube 1990; Lenk 1995). Stattdessen dürfen in unserem Spiel beliebige Paare disjunkter Koalitionen von Spielern fiktive Solidargemeinschaften bilden, um nach den Bestimmungen des Finanzausgleichsgesetzes einen gegenseitigen Finanzausgleich herbeizuführen. Auf diesem Wege können wir mit Hilfe der Spiellösung Progressionseffekte in den gesetzlichen Transferströmen sichtbar machen und den Teilnehmern am Finanzausgleich zurechnen. Diese Effekte machen einen Teil des Konfliktpotentials in der gegenwärtigen Ausgestaltung der föderalen Finanzbeziehungen aus.

Zunächst geben wir im zweiten Abschnitt einen Überblick über die Technik des Länderfinanzausgleichs nach Maßgabe der Finanzausgleichsgesetze von 1993 und 2001. Danach stellen wir in Abschnitt 3 die charakteristische Funktion unseres Finanzausgleichsspiels in allgemeiner Form auf. Anhand elementarer Anforderungen bestimmen wir insbesondere eine geeignete Lösungsvorschrift für das Spiel, die wir zudem in Abschnitt 4 anhand eines stilisierten Beispiels interpretieren. In Abschnitt 5 legen wir die Spiellösung für den Länderfinanzausgleich der Jahre 1998 und 2002 vor. Die Abweichungen der Spielergebnisse von den gesetzlichen Abschöpfungen und Zuweisungen interpretieren wir als eine verdeckte Progression im Länderfinanzausgleich, die sich überwiegend zu Lasten der Geberländer auswirkt. Den Gesamteffekt auf das Transfervolumen beziffern wir in beiden Jahren mit ca. 28\%. Abschnitt 6 beschließt die Arbeit mit einigen Bemerkungen über erste, noch vorläufige Resultate für den Länderfinanzausgleich 2005.

\section{Technik des Länderfinanzausgleichs}

Das Finanzausgleichsgesetz von 1993 bestimmt in $\S 4$, dass der Länderfinanzausgleich in Ausgleichszahlungen der finanzstärkeren Länder an die finanzschwächeren Länder besteht. Ein Land ist gemäß $\S 5$ ausgleichspflichtig oder ein Geberland, wenn seine so genannte Finanzkraftmesszahl seine so genannte Ausgleichsmesszahl 
übersteigt. Im umgekehrten Fall ist das Land ausgleichsberechtigt oder ein Nehmerland. Die Finanzkraftmesszahl des Landes wird im Wesentlichen aus den gesamten Steuereinnahmen dieses Landes und den hälftigen Steuereinnahmen seiner Gemeinden gebildet. Demgegenüber dient die Ausgleichsmesszahl des Landes der Bedarfsermittlung. Hierfür wird die Bevölkerungsgröße des Landes (in den Stadtstaaten Berlin, Bremen und Hamburg zu 135\%) mit der durchschnittlichen Finanzkraft pro Kopf der Gesamtbevölkerung aller Länder multipliziert. Die $\S \S 6-9$ des Finanzausgleichsgesetzes regeln die Berechnung der Finanzkraftmesszahlen und der Ausgleichsmesszahlen im Einzelnen.

Der im Finanzausgleichsgesetz von 1993 angelegte Länderfinanzausgleich schöpft die Überschüsse der Finanzkraft der Geberländer über die jeweiligen Ausgleichsmesszahlen mit einem gebrochen progressiven Tarif ab. Der Tarif beträgt zunächst 15\% (Überschusszone von 100\%-101\%), danach 66\% (Überschusszone von 101\%$110 \%$ ) und zuletzt $80 \%$ (Überschusszone ab 110\%). Im Gegenzug werden die entsprechenden Fehlbeträge der Nehmerländer weitgehend aufgefüllt, und zwar bis zu 92\% der Ausgleichsmesszahlen in voller Höhe und danach zu 37,5\%. Dadurch steigt die Finanzkraft dieser Länder auf mindestens 95\% ihrer Ausgleichsmesszahlen an. Das Gesamtvolumen der Abschöpfungen und Zuweisungen wird mit Hilfe verschiedener so genannter Garantieregelungen harmonisiert, die insbesondere die Finanzkraftreihenfolge unter den Ländern wahren. Die Einzelheiten bestimmt $\S 10$ des Finanzausgleichsgesetzes.

Die abschließende Bemessung der Ausgleichsbeiträge der Geberländer und der Ausgleichszuweisungen an die Nehmerländer nach den Buchstaben des Gesetzes ist aufwändig und zudem algebraisch nicht mehr in geschlossener Form darstellbar. Deshalb haben wir ein Computerprogramm erstellt, das die in den $\S \S 6-10$ veranlassten detaillierten Berechnungen in automatisierter Form durchführt. Hierzu verarbeitet unser Programm Daten der amtlichen Statistik über die Steuereinnahmen und die Bevölkerungsgröße der Länder und ihrer Gemeinden. Auf der Basis dieser Daten reproduziert es für das gesamte Bundesgebiet zuverlässig den relevanten Teil einer umfänglichen Musterrechnung des Bundesverfassungsgerichts für das Jahr 1998 (vgl. BVerfG 1999: Absatz-Nr. 196) und die entsprechenden Teile der endgültigen Abrechnungen des Bundesfinanzministeriums für den Länderfinanzausgleich der Jahre 1998 und 2002 (vgl. BMF 2000, 2004a). ${ }^{1}$ Zudem können wir mit dem Programm den Finanzausgleich zwischen jeder beliebigen Teilmenge von Ländern simulieren.

Im Ergebnis der Neuregelung des bundesstaatlichen Finanzausgleichs durch das Finanzausgleichsgesetz vom 20. Dezember 2001 wird der Finanzausgleich zwischen den Ländern ab dem Jahre 2005 mit einem merklich abgeflachten Ausgleichstarif durchgeführt, der nunmehr sowohl stetig als auch symmetrisch ist. Dafür bezieht das neue Gesetz die Steuerkraft der Gemeinden stärker als bisher in den Finanzausgleich ein. Gleichzeitig wird die kommunale Einwohnerwertung gestrafft. Zudem entfällt

\footnotetext{
${ }^{1}$ Die Kodierung des Länderfinanzausgleichs ist vor allem aufgrund der Garantieregelungen nicht trivial. Exemplarisch zitieren wir das Bundesverfassungsgericht: „Zur sachverständigen Darstellung der die Durchführung des Finanzausgleichsgesetzes bestimmenden Rechenwerke hat sich der Senat der Hilfe des Instituts für Angewandte Mathematik der Universität Karlsruhe . . bedient.“ (BVerfG 1999: AbsatzNr. 196.)
} 
die Mehrzahl der (vergleichsweise ohnehin unbedeutenden) Garantieklauseln, wodurch sich die Bemessung der Ausgleichsbeiträge und -zuweisungen nach $\S 10$ insgesamt erheblich vereinfacht. Erstmals setzt das Finanzausgleichsgesetz von 2001 in $\S 7$ monetäre Anreize in der Form eines Prämiensystems für diejenigen Länder, die eine relativ günstigere Entwicklung der Steuereinnahmen als die Ländergesamtheit verzeichnen. Ansonsten wird die Technik des Finanzausgleichs im Kern beibehalten. Mit einem entsprechend modifizierten Computerprogramm konnten wir bereits eine vorläufige Abrechnung des Bundesfinanzministeriums für den Länderfinanzausgleich des Jahres 2005 nachvollziehen (vgl. BMF 2005).

\section{Spielformulierung}

Die politische Neuregelung des Finanzausgleichs im Rahmen des Solidarpakts II nehmen wir zum Anlass, den Finanzausgleich als ein kooperatives Spiel zu modellieren. In diesem Spiel kann jegliches Paar disjunkter Koalitionen von Bundesländern eine fiktive Solidargemeinschaft gründen und einen zwischenstaatlichen Finanzausgleich nach den Vorgaben des Finanzausgleichsgesetzes herbeiführen (vgl. auch Gottfried/Wiegard 1991). In den jeweiligen Ergebnissen zeichnet sich ab, welche Transfers die Teilnehmer am Ausgleichsverfahren sukzessive je nach Größe und Zusammensetzung einer Solidargemeinschaft auslösen und welche Progression diese Transfers gegebenenfalls aufweisen. Zu diesem Zweck wertet das Spiel alle denkbaren Koalitionspaare aus. Den Verfassungsauftrag zur Schaffung einheitlicher Lebensverhältnisse im gesamten Bundesgebiet (Artikel 106, Absatz 3, Grundgesetz) kleiden wir in die Anforderung, dass in der Spiellösung jedes Bundesland am Finanzausgleich teilnehmen soll.

Hierzu werden wir die Länder im Weiteren von 1 bis $n(=16)$ durchnummerieren und abstrakt als Spieler des kooperativen Spiels bezeichnen. Die Gesamtheit aller Spieler nennen wir $N:=\{1, \ldots, n\}$ und jedes Element der Potenzmenge $2^{N}$ von $N$ eine Koalition. Mit Hilfe unserer Computerprogramme können wir nunmehr für jede Koalition $S \subset N$ der Größe (Kardinalität) $|S|(1 \leq|S| \leq n-1)$ berechnen, welche Ausgleichszuweisung diese Koalition netto im Zuge eines Finanzausgleichs mit einer zufällig ausgewählten disjunkten (und nichtleeren) Koalition $T \subseteq N \backslash S$ erzielen würde, oder welche Ausgleichsverpflichtung gegebenenfalls der Koalition $S$ entstünde. Den Erwartungswert dieses fiktiven Transfers bezeichnen wir als den Transferwert $v(S)$ der Koalition $S$. Demnach besitzt eine finanzschwache Koalition $S$ einen positiven Transferwert. Demgegenüber ist der Transferwert einer finanzstarken Koalition $S$ negativ. Aus Symmetriegründen unterstellen wir, dass jede für den Finanzausgleich mit der Koalition $S$ infrage kommende Koalition $T$ a priori mit derselben statistischen Wahrscheinlichkeit gezogen werden kann. In diesem Fall entspricht $v(S)$ dem arithmetischen Mittel der Transfers zwischen der Koalition $S$ und den $2^{n-|S|}-1$ (nichtleeren) Koalitionen $T \subseteq N \backslash S$. Insbesondere hat somit die große Koalition aller Spieler $N$ den Wert $v(N)=0$. Weiterhin setzen wir zur Vereinfachung der Notation $v(\emptyset)=0$. Demnach ist $v: 2^{N} \rightarrow \mathbb{R}$ die charakteristische Funktion des angenommenen Finanzausgleichsspiels $\Gamma:=(N, v)$ (vgl. Weber 1994). 
Unter einer Lösung dieses Spiels verstehen wir eine Vorschrift $\varphi: \Gamma \rightarrow \mathbb{R}^{n}$, die dem Spiel (genau) ein Element $\mathbf{x}:=\left(x_{1}, \ldots, x_{n}\right)$ der Pre-Imputationsmenge $I(\Gamma):=\left\{\mathbf{x} \in \mathbb{R}_{+}^{n}: \sum_{i \in N} x_{i}=0\right\}$ zuordnet. Materiell bestimmt diese Vorschrift für jeden Spieler (jedes Bundesland) $i$ in der Regel einen Ausgleichsbeitrag $x_{i}<0$ oder eine Ausgleichszuweisung $x_{i}>0$. Auf diese Weise werden alle Spieler am Finanzausgleich beteiligt. Im Grenzfall kann $x_{i}$ auch zufällig den Wert Null annehmen.

Mit der Ausgestaltung einer konkreten Vorschrift $\varphi$ verknüpfen wir den grundlegenden Anspruch, dass die Ausgleichsbeiträge oder -zuweisungen nach der fiskalischen Leistungsfähigkeit der Spieler bemessen werden. Deshalb verlangen wir zunächst, dass zwei beliebige symmetrische Spieler $i$ und $j$ mit jeweils denselben Finanzkraftmesszahlen und Ausgleichsmesszahlen in der Spiellösung gleichbehandelt werden. In diesem Sinne soll die Spiellösung anonym sein. Wir können die Spieler dann beliebig bezeichnen oder reihen:

Gleichbehandlung: Sei $v(S \cup\{i\})=v(S \cup\{j\})$ für alle Koalitionen $S \subseteq N \backslash\{i, j\}$. Dann gelte $\varphi_{i}(N, v)=\varphi_{j}(N, v)$.

Weiterhin postulieren wir, dass die Ausgleichsverpflichtung eines beliebigen Spielers $i$ nicht erhöht wird oder gegebenenfalls seine Ausgleichszuweisung nicht sinkt, falls sich die Finanzkraft dieses Spielers im Vergleich zur Finanzkraft aller übrigen Spieler ceteris paribus verschlechtert. Dieser Umstand wird in der charakteristischen Funktion des Spiels dadurch angezeigt, dass die Transferwerte aller Koalitionen steigen, denen der Spieler $i$ angehört. Demnach können wir unsere Bedingung wie folgt präzisieren:

Monotonie: Für zwei beliebige charakteristische Funktionen $v$ und $v^{\prime}$ des Finanzausgleichsspiels und einen beliebigen Spieler $i \in N$ sei $v(S \cup\{i\})-v(S) \geq v^{\prime}(S \cup$ $\{i\})-v^{\prime}(S)$ für alle Koalitionen $S \subseteq N \backslash\{i\}$. Dann gelte $\varphi_{i}(N, v) \geq \varphi_{i}\left(N, v^{\prime}\right)$.

Unsere Anforderungen stellen bereits eine vollständige Axiomatisierung der gesuchten Spiellösung $\varphi$ dar (vgl. Young 1985: 70-71; Peleg/Sudhölter 2003: 210-211): Theorem: Sei $\varphi$ eine Lösung des Finanzausgleichsspiels $\Gamma$, die dem Spiel eindeutig ein Element $\mathbf{x} \in \mathcal{I}(\Gamma)$ zuordnet und die den Anforderungen der Gleichbehandlung und der Monotonie genügt. Dann ist $\varphi$ der Shapley-Wert

$$
\varphi_{i}:=\sum_{S \subseteq N, i \in S} \frac{(|S|-1) !(n-|S|) !}{n !}[v(S)-v(s \backslash\{i\})] \quad \text { für alle } i \in N
$$

dieses Spiels.

Der Shapley-Wert (eigentlich: Shapley-Vektor) weist jedem Spieler $i$ denjenigen Geldbetrag $x_{i}$ zu, den der Spieler bei der Koalitionsbildung im Mittel als Ausgleichsverpflichtung oder -zuweisung auslöst. ${ }^{2}$ Insoweit ist die Spiellösung marginalistisch oder utilitaristisch geprägt (vgl. Moulin 1988: 107; Young 1994: 200). ${ }^{3}$

\footnotetext{
${ }^{2}$ Rechnerisch werden hierzu die gewogenen Grenzbeiträge $v(S)-v(S \backslash\{i\})$ des jeweiligen Spielers $i$ über alle Koalitionen kumuliert, denen der Spieler angehört. Bei $n$ Spielern gibt es insgesamt $\left(\begin{array}{c}n-1 \\ |S|-1\end{array}\right)$ Koalitionen der Größe $|S|$, die den Spieler $i$ enthalten. Überdies bestehen $n$ Möglichkeiten für die Auswahl des letzten Mitglieds einer Koalition. Deshalb hat der Grenzbeitrag des Spielers $i$ in der jeweiligen Koalition $S$ das Gewicht $\frac{1}{n\left(\begin{array}{c}n-1 \\ |S|-1\end{array}\right)}=\frac{(|S|-1) !(n-|S|) !}{n(n-1) !}=\frac{(|S|-1) !(n-|S|) !}{n !}$.

${ }^{3}$ Aus diesem Grund erhalten wir insbesondere das Resultat $\left(x_{1}, \ldots, x_{n}\right)=(0, \ldots, 0)$, falls im theoretischen Grenzfall alle Spieler gleich (d.h. Strohmänner) sind und folglich jede Koalition $S \subseteq N$ denselben Wert $v(S)=0$ hat.
} 


\section{Interpretation}

Bevor wir unser Finanzausgleichsspiel für die deutschen Bundesländer lösen, wollen wir der grundsätzlichen Frage nachgehen, wie die Spielergebnisse auszulegen sind. Hierzu betrachten wir das folgende stilisierte Beispiel eines Finanzausgleichs zwischen drei Regionen. In diesem Beispiel habe Region 1 die Finanzkraft 36. Der Einfachheit halber nehmen wir an, dass die Regionen 2 und 3 identisch sind und dass ihre Finanzkraft zudem jeweils 0 beträgt. Im Übrigen sei die Einwohnerschaft in allen Regionen gleich groß, so dass wir den Einfluss der Bevölkerungsstruktur auf den Finanzausgleich vernachlässigen können. Der Gesetzgeber verlange die vollständige Elimination aller Finanzkraftunterschiede. Dann ist $\frac{1}{3}(36+0+0)=12$ die anzuwendende Ausgleichsmesszahl. Demnach lautet der Vektor der Abschöpfungen (-) und Zuweisungen $(+)$ im gesetzlichen Finanzausgleich $(-24,12,12)$.

Im zugehörigen Finanzausgleichsspiel mit der Spielermenge $N=\{1,2,3\}$ wenden wir die Technik des gesetzlichen Finanzausgleichs sinngemäß an. Zunächst berücksichtigen wir, dass der solidarische Spieler 1 einen paarweisen Finanzausgleich mit dem Spieler 2 oder mit dem Spieler 3 vereinbaren kann. In beiden Fällen geht der Spieler 1 dieselbe gesetzliche Ausgleichsverpflichtung in Höhe von jeweils -18 ein. Ebenso kann der Spieler 1 in die angestrebte Bildung der großen Koalition aller drei Spieler einwilligen. In diesem weitergehenden dritten Fall beträgt seine gesetzliche Ausgleichsverpflichtung bereits -24 . Sie ist das Resultat einer Verbreiterung der Bemessungsgrundlage in der größeren Solidargemeinschaft. In der Folge tendiert die mittlere gesetzliche Ausgleichsverpflichtung des Spielers 1 von vormals $\frac{1}{2}[(-18)+(-18)]=-18$ gegen den neuen, endgültigen Wert $\frac{1}{3}[(-18)+(-18)+$ $(-24)]=-20$, den wir als Transferwert $v(\{1\})$ bezeichnen. Für die übrigen Spieler und die Koalitionen von zwei Spielern überlegen wir entsprechend. Die komplettierte charakteristische Funktion des Spiels lautet:

\begin{tabular}{|c|c|c|c|c|c|c|c|c|}
\hline$S$ & $\emptyset$ & $\{1\}$ & $\{2\}$ & $\{3\}$ & $\{1,2\}$ & $\{1,3\}$ & $\{2,3\}$ & $N$ \\
\hline$v(\{S\})$ & 0 & -20 & 10 & 10 & -12 & -12 & 24 & 0 \\
\hline
\end{tabular}

Nun stellen wir die Berechnungsformel für den Shapley-Wert des Spielers 1 auf. Hierfür ziehen wir (1) heran und setzen darin vorübergehend $n=3$ :

$$
\begin{aligned}
\varphi_{1}= & \frac{1}{3}[v(\{1\})-v(\emptyset)] \\
& +\frac{1}{6}[v(\{1,2\})-v(\{2\})]+\frac{1}{6}[v(\{1,3\})-v(\{3\})] \\
& +\frac{1}{3}[v(\{1,2,3\})-v(\{2,3\})] .
\end{aligned}
$$

Aus (2) gewinnen wir wegen $v(\emptyset)=v(N)=0$ und vermöge der Identitäten

$$
\begin{aligned}
v(\{1\})+v(\{2\})+v(\{3\}) & \equiv 0 \\
v(\{1,2\})+v(\{1,3\})+v(\{2,3\}) & \equiv 0
\end{aligned}
$$


den algebraischen Ausdruck

$$
\varphi_{1}=-v(\{2,3\})+\frac{1}{2}[v(\{2,3\})+v(\{1\})] .
$$

Seine Auswertung anhand der charakteristischen Funktion ergibt $\varphi_{1}=-22$. Da die Spieler 2 und 3 identisch sind, erhalten wir auch sogleich die vollständige Spiellösung $\left(\varphi_{1}, \varphi_{2}, \varphi_{3}\right)=(-22,11,11)$.

Wir konstatieren, dass die Spiellösung den Spieler 1 von einem Teil seiner gesetzlichen Ausgleichsverpflichtung entbindet. Die Minderung wird den ausgleichsberechtigten Spielern 2 und 3 zu gleichen Teilen als gemeinsames Opfer angelastet. Unsere Beziehung (5) bildet das Opfer anschaulich als hälftige Lücke $\frac{1}{2}[v(\{2,3\})+$ $v(\{1\})]=2$ zwischen den Transferwerten der Koalitionen $\{2,3\}$ und $\{1\}$ ab. Materiell gibt der jeweilige Fehlbetrag zugleich den (absoluten) Anstieg $\frac{1}{2}[(-18)+(-18)]-$ $v(\{1\})=2$ der mittleren gesetzlichen Ausgleichsverpflichtung des Spielers 1 im Zuge des Aufbaus der großen Koalition wieder. ${ }^{4}$ Im Umfang dieses Effektes verlangt die Spiellösung den Spielern 2 und 3 ein kooperatives Zugeständnis ab.

Mithin können wir unser Finanzausgleichsspiel für die deutschen Bundesländer angemessen interpretieren: Das Spiel schlüsselt gegebenenfalls auf, wie weitgehend die gesetzlichen Abschöpfungen und Zuweisungen einer verdeckten Progression unterliegen, die von den Teilnehmern am Ausgleichsverfahren je nach deren Finanzkraft ausgelöst wird. Die Länderstruktur der Progression wird im Verlaufe eines Abgleichs der 16 Shapley-Werte mit ihren empirischen Referenzgrößen sichtbar. Ein gesamthaftes Resultat für das globale Transfervolumen im Finanzausgleich erhält man hernach durch Aggregation.

\section{Spielergebnisse}

Auf der Basis der empirischen Eingangsdaten des Länderfinanzausgleichs 1998 (vgl. BMF 2000) konnten wir die zugehörige charakteristische Funktion unseres Finanzausgleichsspiels mittels eines leistungsfähigen Personal Computers (Pentium 4, $2.40 \mathrm{GHz}$ ) und eines Aufwands von etwa zweieinhalb Stunden Rechenzeit erzeugen. Für die Bestimmung des zu dieser Funktion gehörenden Shapley-Wertes mit Hilfe der Software CG_SOL von Derks/Kuipers (1997) benötigten wir weniger als eine Sekunde Rechenzeit. Tabelle 1 gibt einerseits die tatsächlichen Transfers im Länderfinanzausgleich 1998 gemäß dem Finanzausgleichsgesetz von 1993 (Spalte FAG) sowie andererseits den Shapley-Wert unseres entsprechenden Finanzausgleichsspiels (Spalte FA-Spiel) wieder. Die jeweiligen Angaben werden in Abbildung 1 graphisch illustriert. Tabelle 1 zeigt in ihrer letzten Spalte zudem die Progressionseffekte in Gestalt der relativen Abweichung des Shapley-Wertes von den jeweiligen empirischen Vergleichsdaten $(=100 \%)$ an. Wir haben die Länder in der für die Berichterstattung des Bundesfinanzministeriums üblichen Abfolge aufgelistet.

\footnotetext{
${ }^{4}$ Diese Aussage bestätigt man allgemein wie folgt: Seien $x_{1}$ und $x_{2}$ die Ausgleichsverpflichtungen des Spielers 1 im paarweisen Finanzausgleich mit dem Spieler 2 bzw. 3. (Im Beispiel: $x_{1}=x_{2}=-18$.) Dann gilt $v(\{1\})=\frac{1}{3}\left(x_{1}+x_{2}-v(\{2,3\})\right.$. Beidseitige Addition von $v(\{2,3\})$ und anschließende Division durch 2 ergeben $\frac{1}{2}[v(\{2,3\})+v(\{1\})]=\frac{1}{6}\left(x_{1}+x_{2}\right)+\frac{1}{3} v(\{2,3\})=\frac{1}{2}\left(x_{1}+x_{2}\right)-v(\{1\})$.
} 
Tabelle 1 Ausgleichsbeiträge (-) und -zuweisungen (+) 1998 (in 1000 Euro)

\begin{tabular}{lrrr}
\hline Bundesland & \multicolumn{1}{c}{ FAG } & \multicolumn{1}{c}{ FA-Spiel } & \multicolumn{1}{c}{ Progression } \\
\hline Nordrhein-Westfalen (NW) & -1.583 .020 & -1.113 .594 & $29,65 \%$ \\
Bayern (BY) & -1.486 .500 & -1.080 .784 & $27,29 \%$ \\
Baden-Württemberg (BW) & -1.777 .899 & -1.272 .646 & $28,42 \%$ \\
Niedersachsen (NI) & 402.864 & 285.622 & $-29,10 \%$ \\
Hessen (HE) & -1.758 .195 & -1.256 .642 & $28,53 \%$ \\
Sachsen (SN) & 1.019 .426 & 721.414 & $-29,23 \%$ \\
Rheinland-Pfalz (RP) & 219.396 & 161.757 & $-26,27 \%$ \\
Sachsen-Anhalt (ST) & 617.263 & 442.980 & $-28,23 \%$ \\
Schleswig-Holstein (SH) & -53 & -13.672 & $-25.696,23 \%$ \\
Thüringen (TH) & 595.098 & 428.190 & $-28,05 \%$ \\
Brandenburg (BB) & 533.996 & 382.956 & $-28,28 \%$ \\
Mecklenburg-Vorpommern (MV) & 448.465 & 324.399 & $-27,66 \%$ \\
Saarland (SL) & 116.388 & 85.906 & $-26,19 \%$ \\
Berlin (BE) & 2.500 .686 & 1.800 .258 & $-28,01 \%$ \\
Hansestadt Hamburg (HH) & -314.315 & -239.970 & $23,65 \%$ \\
Hansestadt Bremen (HB) & 466.399 & 343.826 & $-26,28 \%$ \\
\hline
\end{tabular}

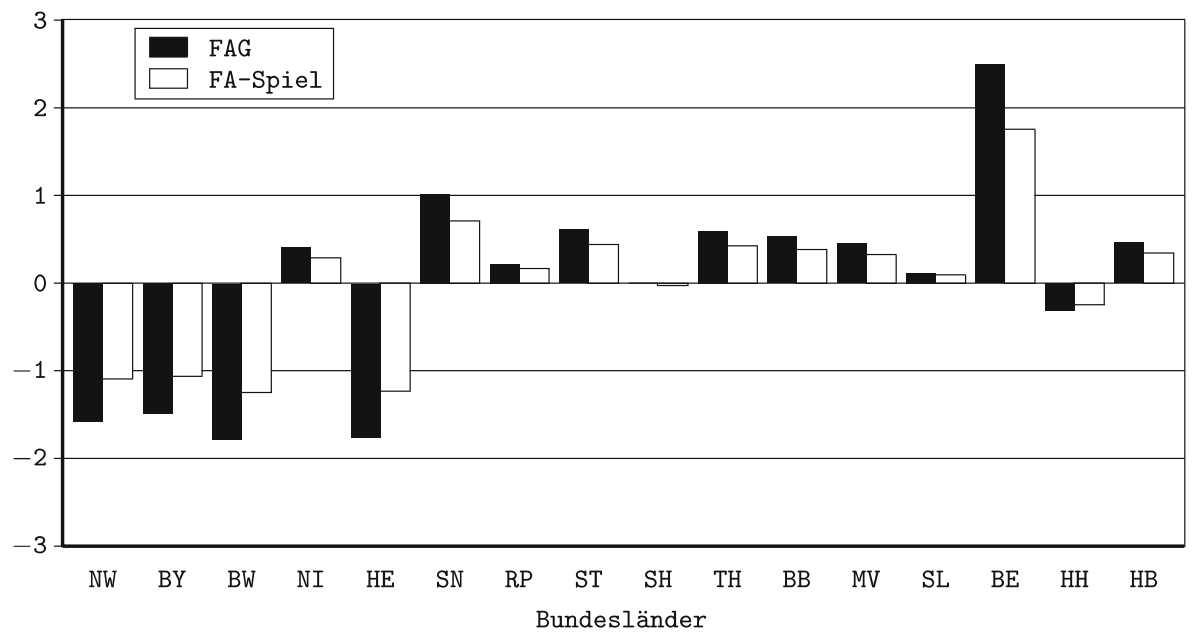

Abb. 1 Ausgleichsbeiträge (-) und -zuweisungen (+) 1998 (in Mrd. Euro)

Der Shapley-Wert unseres Finanzausgleichsspiels spiegelt zunächst die Ergebnisse des gesetzlichen Finanzausgleichs wider. Er weist die Länder BadenWürttemberg, Bayern, Hamburg, Hessen, Nordrhein-Westfalen und SchleswigHolstein als Geberländer im Finanzausgleich aus. Im Einzelnen würden die Länder Baden-Württemberg, Bayern, Hessen und Nordrhein-Westfalen zu bedeutenden Ausgleichszahlungen herangezogen. Die Länder Hamburg und Schleswig-Holstein würden die restlichen Ausgleichsverpflichtungen übernehmen. Alle übrigen Länder erlangen in der Spiellösung den Status von Ausgleichsempfängern. Die bei weitem größte Ausgleichszuweisung erhielte demnach das Land Berlin. Insoweit führt unser Spiel erwartungsgemäß zu denselben qualitativen Resultaten wie der gesetzliche Länderfinanzausgleich 1998. 
Tabelle 2 Ausgleichsbeiträge (-) und -zuweisungen (+) 2002 (in 1000 Euro)

\begin{tabular}{lrrr}
\hline Bundesland & \multicolumn{1}{c}{ FAG } & \multicolumn{1}{c}{ FA-Spiel } & Progression \\
\hline Nordrhein-Westfalen (NW) & -1.626 .901 & -1.143 .578 & $29,71 \%$ \\
Bayern (BY) & -2.038 .189 & -1.463 .283 & $28,21 \%$ \\
Baden-Württemberg (BW) & -1.639 .891 & -1.199 .698 & $26,84 \%$ \\
Niedersachsen (NI) & 485.966 & 363.154 & $-25,27 \%$ \\
Hessen (HE) & -1.903 .857 & -1.379 .595 & $27,54 \%$ \\
Sachsen (SN) & 1.036 .130 & 733.700 & $-29,19 \%$ \\
Rheinland-Pfalz (RP) & 416.527 & 301.552 & $-27,60 \%$ \\
Sachsen-Anhalt (ST) & 600.462 & 430.811 & $-28,25 \%$ \\
Schleswig-Holstein (SH) & 111.344 & 77.364 & $-30,52 \%$ \\
Thüringen (TH) & 565.086 & 405.971 & $-28,16 \%$ \\
Brandenburg (BB) & 534.095 & 382.465 & $-28,39 \%$ \\
Mecklenburg-Vorpommern (MV) & 434.821 & 314.114 & $-27,76 \%$ \\
Saarland (SL) & 137.797 & 100.384 & $-27,15 \%$ \\
Berlin (BE) & 2.669 .574 & 1.922 .548 & $-27,98 \%$ \\
Hansestadt Hamburg (HH) & -189.732 & -144.923 & $23,62 \%$ \\
Hansestadt Bremen (HB) & 406.768 & 299.014 & $-26,49 \%$ \\
\hline
\end{tabular}

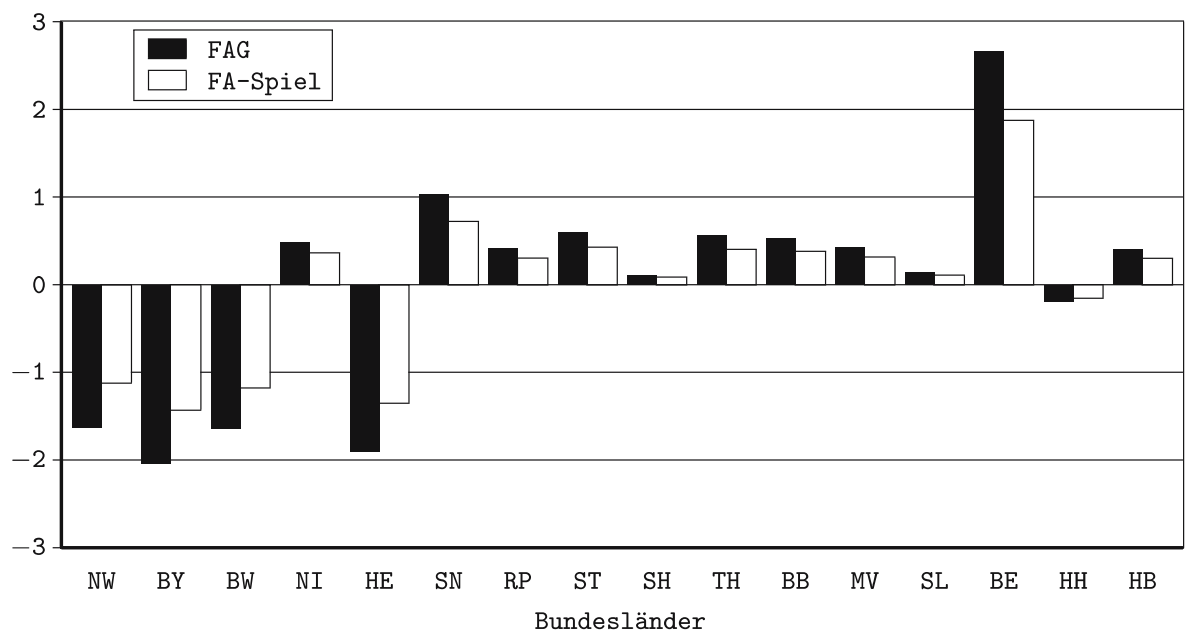

Abb. 2 Ausgleichsbeiträge (-) und -zuweisungen (+) 2002 (in Mrd. Euro)

Gleichwohl fällt das Transfervolumen (Summe der Ausgleichszuweisungen) im Spielergebnis (4.977.308 Tsd. Euro) um immerhin 28,07\% geringer als im gesetzlichen Länderfinanzausgleich 1998 (6.919.981 Tsd. Euro) aus. Wir sehen hierin einen globalen Indikator für die Progression der gesetzlichen Transfers. Von einer Bereinigung dieser Transfers um die anteiligen länderspezifischen Progessionseffekte würde vor allem das Land Nordrhein-Westfalen mit einem Rückggang seines Ausgleichsbeitrages um 29,65\% gegenüber dem empirischen Referenzwert profitieren. Aber auch die Geberländer Baden-Württemberg, Bayern, Hamburg und Hessen könnten mindestens 23,65\% (Hamburg) und höchstens 28,53\% (Hessen) ihrer Ausgleichsbeiträge einsparen. Im Gegenzug müssten alle Nehmerländer finanzielle Einbußen im Umfang zwischen 26,19\% (Saarland) und 29,23\% (Sachsen) der ihnen 1998 gewährten Ausgleichszuweisungen hinnehmen. Ein Sonderfall ist 
Schleswig-Holstein, dessen Ausgleichsverpflichtung zunähme. Das deutet darauf hin, dass dieses Land von der Progression der Ausgleichslasten der übrigen Geberländer begünstigt wird.

In einer weiteren Modellrechnung haben wir aus den empirischen Eingangsdaten des Länderfinanzausgleichs 2002 (vgl. BMF 2004a) erneut die entsprechende charakteristische Funktion unseres Finanzausgleichsspiels generiert und den zugehörigen Shapley-Wert berechnet. Die Resultate in Tabelle 2 und in Abbildung 2 zeigen wie erwartet, dass der Shapley-Wert wiederum allen faktischen Geberländern - im Jahr 2002 die Länder Baden-Württemberg, Bayern, Hamburg, Hessen und NordrheinWestfalen - Ausgleichsbeiträge in unterschiedlicher Höhe aufbürdet. Die übrigen Länder würden ebenso wie im gesetzlichen Länderfinanzausgleich 2002 Ausgleichszuweisungen beziehen.

Abermals liegt das Transfervolumen in der Spiellösung (5.331.077 Tsd. Euro) deutlich unter dem tatsächlichen Transfervolumen (7.398.570 Tsd. Euro). Die implizierte globale Progression der gesetzlichen Transfers beträgt 27,94\%. Ohne die Progression könnte vor allem das Land Nordrhein-Westfalen seinen Ausgleichsbeitrag um 29,71\% des empirischen Referenzwerts im Jahr 2002 verringern. Die Einsparungen der anderen Geberländer würden 23,62\% (Hamburg) - 28,21\% (Bayern) der empirischen Vergleichswerte ausmachen. Hierzu müssten die Nehmerländer auf Ausgleichszuweisungen im Ausmaß zwischen 25,27\% (Niedersachsen) und 30,52\% (Schleswig-Holstein) der Zuweisungen im Vergleichsjahr 2002 verzichten.

Die Frage liegt nahe, wie weitgehend die Spielergebnisse vom Verlauf des Abschöpfungstarifs in $\S 10$ des Finanzausgleichsgesetzes abhängen. Insbesondere wäre zu prüfen, welchen Einfluss die Abflachung dieses Tarifs im Zuge der Neuregelung des horizontalen Finanzausgleichs für den Zeitraum 2005-2019 auf unsere Resultate hat. Mit einem Ausblick auf vorliegende erste Daten des Bundesfinanzministeriums für den Länderfinanzausgleich 2005 beschließen wir unsere Ausführungen.

\section{Die neue Finanzverteilung}

In dieser Arbeit wird der so genannte horizontale Finanzausgleich zwischen den Ländern der Bundesrepublik Deutschland in einem kooperativen Spiel abgebildet. Darin treten die Bundesländer als Spieler auf. Insbesondere kann jedes Paar disjunkter Koalitionen von Spielern eine fiktive Solidargemeinschaft bilden und nach den Bestimmungen des Finanzausgleichsgesetzes einen gegenseitigen Finanzausgleich herbeiführen. Mithin zeigt sich, welche Transfers die Teilnehmer am Finanzausgleich je nach Größe und Zusammensetzung einer Solidargemeinschaft auslösen. Hierzu wertet die charakteristische Funktion des Spiels alle denkbaren Koalitionspaare aus. Auf dieser Basis konnten wir in der Spiellösung hypothetische Ausgleichsbeiträge der jeweiligen Geberländer und Ausgleichszuweisungen an die betreffenden Nehmerländer für den Finanzausgleich der Jahre 1998 und 2002 gewinnen. Die Abweichungen der Spielergebnisse von den gesetzlichen Abschöpfungen und Zuweisungen haben wir als eine verdeckte Progression im Länderfinanzausgleich interpretiert, die sich überwiegend zu Lasten der Geberländer auswirkt. Der da- 
mit verbundene Gesamteffekt auf das Transfervolumen beträgt in beiden Jahren ca. $28 \%$

Die Sensitivität unserer Resultate in Bezug auf den gesetzlichen Abschöpfungstarif können wir bereits anhand einer Abrechnung des Bundesfinanzministeriums für den Länderfinanzausgleich 2005 (vgl. BMF 2005) überprüfen. Diese noch vorläufige Abrechnung wendet erstmals das neue Finanzausgleichsgesetz vom 20. Dezember 2001 an. In dem vorliegenden Zahlenwerk zeichnet sich ab, dass vor allem die Geberländer Bayern, Baden-Württemberg und Hessen absehbar unverändert hohe Ausgleichsbeiträge leisten werden. Für das Bundesland Berlin setzt das Bundesfinanzministerium wiederum die weitaus höchste Ausgleichszuweisung (2.440.596 Tsd. Euro) an, die jedoch um 9,70\% unter dem Vorjahreswert (2.702.879 Tsd. Euro) liegt. Dafür dürfen insbesondere die anderen ostdeutschen Bundesländer mit höheren Ausgleichszuweisungen als im Vorjahr rechnen. In der Folge übertrifft das voraussichtliche Transfervolumen des Jahres 2005 (6.884.636 Tsd. Euro) den Vergleichswert des Vorjahres 2004 (6.804.839 Tsd. Euro) noch um 1,17\%.

Unsere einstweiligen Spielergebnisse für dieses Jahr unterscheiden sich qualitativ wenig von den Resultaten für die Jahre 1998 und 2002. Die Progression im globalen Transfervolumen sinkt nur geringfügig unter die 28\%-Marke. Daraus schließen wir, dass die Progression vor allem durch die Mechanik der Ausgleichsmesszahlen determiniert wird. Diese Form der Ausgestaltung des Finanzausgleichs zwischen 16 heterogenen Bundesländern stößt nachweislich an die Grenze der politischen Akzeptanz. Alternativ könnte man eine exogene Anspruchsnorm für die Bemessung von Ausgleichsbeiträgen definieren und diese Norm zum Beispiel nur noch über mittlere Zeiträume den jeweiligen Erfordernissen anpassen. Hierdurch würde im Übrigen der Stellenwert besonderer Anreizmechanismen für den Finanzausgleich wie zum Beispiel eine Prämienregelung relativiert.

\section{Literatur}

Buhl, H.U. (1986): Ein finanzwissenschaftlich-methodischer Diskussionsbeitrag zur Neuordnung des Länderfinanzausgleichs. Finanzarchiv 44, 476-483

Buhl, H.U., Pfingsten, A. (1986): Eigenschaften und Verfahren für einen angemessenen Länderfinanzausgleich in der Bundesrepublik Deutschland. Finanzarchiv 44, 98-109

Buhl, H.U., Pfingsten, A. (1991): Zehn Gebote für Finanzausgleichsverfahren und ihre Implikationen. Wirtschaftsdienst 9, 481-484

BMF/Bundesministerium der Finanzen (2000): Zweite Verordnung zur Durchführung des Finanzausgleichsgesetzes im Ausgleichsjahr 1998. Bundesrat: Drucksache 123/00

BMF/Bundesministerium der Finanzen (2004a): Zweite Verordnung zur Durchführung des Finanzausgleichsgesetzes im Ausgleichsjahr 2002. Bundesrat: Drucksache 34/02

BMF/Bundesministerium der Finanzen, Hrsg. (2004b): Dokumentation Bund-Länder Finanzbeziehungen auf der Grundlage der geltenden Finanzverfassungsordnung. 6. Aufl., BMF: Berlin

BMF/Bundesministerium der Finanzen (2005): Vorläufige Abrechnung des Länderfinanzausgleichs für das Ausgleichsjahr 2005. BMF: Berlin

BVerfG/Bundesverfassungsgericht (1999): 2 BvF 2/98 vom 11.11.1999

Derks, J., Kuipers, J. (1997): CG_SOL. http://www.math.unimaas.nl/personal/jeand/home1.htm [17.6.2006]

Gottfried, P., Wiegard, W. (1991): Finanzausgleich nach der Vereinigung: Gewinner sind die alten Länder. Wirtschaftsdienst 71, 453-461

Lenk, T. (1995): Vergleich alternativer Ausgleichsmechanismen für den Länderfinanzausgleich. Zeitschrift für Wirtschafts- und Sozialwissenschaften 115, 231-273 
Moulin, H. (1988): Axioms of Cooperative Decision Making. New York: Cambridge University Press Peleg, B., Sudhölter, P. (2003): Introduction to the Theory of Cooperative Games. Boston, Kluwer Academic Publishers

Shapley, L.S. (1953): A Value for n-Person Games. In: H.W. Kuhn und A.W. Tucker, Hrsg., Contributions to the Theory of Games. Vol. II, Annals of Mathematics Studies 28, 307-317

Taube, R. (1990): Ein Vorschlag zur Reform des Länderfinanzausgleichs. Wirtschaftsdienst 7, 372-380

Weber, R.J. (1994): Games in Coalitional Form. In: R.J. Aumann und S. Hart, Hrsg., Handbook of Game Theory with Economic Applications. Vol. II, Amsterdam: North-Holland, 1285-1303

Winter, E. (2002): The Shapley Value. In: R.J. Aumann und S. Hart, Hrsg., Handbook of Game Theory with Economic Applications. Vol. III, Amsterdam: North-Holland, 2025-2054

Young, H.P. (1985): Monotonic Solutions of Cooperative Games. International Journal of Game Theory 14, 65-72

Young, H.P. (1994): Equity: In Theory and Practice. Princeton/NJ: Princeton University Press 